\title{
Adaptive Parameter Identification Based on Morlet Wavelet and Application in Gearbox Fault Feature Detection
}

\author{
Shibin Wang, Z. K. Zhu, Yingping He, and Weiguo Huang \\ School of Urban Rail Transportation, Soochow University, Suzhou 215006, China \\ Correspondence should be addressed to Z. K. Zhu, zkzhu@ustc.edu
}

Received 26 July 2010; Accepted 22 October 2010

Academic Editor: T.-H. Li

Copyright () 2010 Shibin Wang et al. This is an open access article distributed under the Creative Commons Attribution License, which permits unrestricted use, distribution, and reproduction in any medium, provided the original work is properly cited.

Localized defects in rotating mechanical parts tend to result in impulse response in vibration signal, which contain important information about system dynamics being analyzed. Thus, parameter identification of impulse response provides a potential approach for localized fault diagnosis. A method combining the Morlet wavelet and correlation filtering, named Cyclic Morlet Wavelet Correlation Filtering (CMWCF), is proposed for identifying both parameters of impulse response and the cyclic period between adjacent impulses. Simulation study concerning cyclic impulse response signal with different SNR shows that CMWCF is effective in identifying the impulse response parameters and the cyclic period. Applications in parameter identification of gearbox vibration signal for localized fault diagnosis show that CMWCF is effective in identifying the parameters and thus provides a feature detection method for gearbox fault diagnosis.

\section{Introduction}

Rotating machines play an important role in many industrial applications, such as aircraft engines, automotive transmission systems, and wind power generation. Most of the machinery was operated by means of gears and other rotating parts, which may develop faults. The study of fault diagnosis of rotating machine by fault feature detection from vibration signals has thus attracted more and more attention over the past decade.

Gearbox, as an important mechanism for transmitting power or rotation, is widely used in industrial applications. The occurrence of impulse response in gearbox vibration signals usually means that there exist mechanical defects or faults. Most gear faults are due to gear damage, such as tooth wear, cracks, scoring, spalling, chipping, and pitting $[1,2]$. With such flaws existing on gears, progressive damage will occur and ultimately result in gear tooth breakage, which may cause significant economic loss. For gearbox fault diagnosis, therefore, it is very important to extract the information of impulse response from vibration signals.

So far, different techniques have been proposed to analyze the vibration signal for fault diagnosis, such as timefrequency/time-scale methods, empirical mode decomposi- tion (EMD), and matching pursuit (MP). Time-frequency distribution is a three-dimensional time, frequency, and amplitude representation of a signal, which is commonly used to diagnose faults in mechanical systems because the time-frequency distribution can accurately extract the desired frequencies from a nonstationary signal [3-5]. The time-frequency distributions are linear or bilinear. The former includes the short time Fourier transform, which provides constant resolution for all frequency since it uses the same window for the analysis of the entire signal. The latter includes the Wigner-Ville distribution, the Choi-Williams distribution, and improved ones. There is no doubt that the Wigner-Ville distribution has good concentration in the time-frequency plane. However, even if support areas of the signal do not overlap each other, interference terms will appear to mislead the signal analysis. Time-scale methods often refer to wavelet transform. In wavelet analysis, a signal is analyzed at different scales or resolution: larger time and smaller scale window is used to look at the approximate stationarity of the signal and smaller time and larger scale window at transients. Reference [6] summarizes the application of the wavelet in machine fault diagnosis, including the fault feature extraction, the denoising and extraction of the weak signals, and the system identification. 
EMD is an adaptive decomposition method proposed by Huang et al. [7], which in essence extracts the intrinsic oscillation of the signal being analyzed through their characteristic time scales (i.e., local properties of the signal itself) and decomposes the signal into a number of intrinsic mode functions (IMFs), with each IMF corresponding to a specific range of frequency components contained within the signal. Because it still has some shortcomings when it comes to calculating instantaneous frequency [8] or in some cases it may reveal plausible characteristics due to the mode mixing $[9,10]$, it is untenable in effective application in impulse detection and analysis.

Matching pursuit algorithm, a greedy algorithm that chooses a waveform that is the most adapted to approximate part of the signal at each iteration, is effective in analyzing impulse response signals; however, the excessive computational cost limits its engineering applications [11]. Correlation filtering, enlightened from matching pursuit, is used based on Laplace wavelet to identify the parameters of impulse response by calculating the maximal correlation value, which is employed by Freudinger et al. to identify the modal parameters of a flutter for aerodynamic and structural testing [12]. Similar efforts were made by $\mathrm{Zi}$ et al. for the identification of the natural frequency of a hydrogenerator shaft and the wear fault diagnosis of the intake valve of an internal combustion engine [13]. Qi et al. employed Laplace wavelet correlation filtering together with empirical mode decomposition to identify modal parameters [14]. An integrated approach, consisting of empirical mode decomposition, Laplace wavelet correlation filtering, and wavelet finite element model, proposed by Dong et al. for rotor crack detection, was effective in identifying the position and the depth of different cracks [15].

Laplace wavelet correlation filtering is effective in detecting a single transient impulse response. However, localized defects in rotating mechanical parts tend to result in multiple impulse responses, which are generally cyclic impulse responses. Considering that the waveform of Morlet wavelet is in shape similar to transient vibration caused by gearbox localized defects $[16,17]$ and cyclostationarity matches the key feature of the gearbox vibration $[18,19]$, Cyclic Morlet Wavelet Correlation Filtering (CMWCF) is thus proposed, which, based on correlation filtering, constructs the cyclic Morlet wavelet and identify both the impulse response parameters and the cyclic period for diagnosed gear fault.

The remainder of the paper is organized as follows. In Section 2, the basic theoretical background concerning CMWCF is introduced. Section 3 gives a simulation study and analysis to verify the proposed method. Section 4 applies the method in gearbox transient feature detection by parameter identification for fault diagnosis. Finally, conclusions are drawn in Section 5.

\section{Adaptive Parameter Identification Based on Morlet Wavelet}

In this section, a method of adaptive parameter identification of Morlet wavelet based on correlation filtering is presented. Using correlation filtering, the parameters of Morlet wavelet are firstly identified to detect the impulse response. Secondly, cyclic Morlet wavelet is constructed to detect the cyclic period between adjacent impulse responses. The proposed method is suitable for not only identifying the parameters of the impulse response but also detecting the cyclic period.

2.1. Morlet Wavelet and Parametric Representation. Morlet wavelet is one of the most popular nonorthogonal wavelets, defined in the time domain as a harmonic wave multiplied by a Gaussian time domain window:

$$
\psi_{\text {Morlet }}(t)=\exp \left(\frac{-\beta^{2} t^{2}}{2}\right) \cos (\pi t) .
$$

Morlet wavelet is a cosine signal that decays exponentially on both the left and the right sides. This feature makes it very similar to an impulse. It has been used for impulse isolation and mechanical fault diagnosis $[16,17]$.

The parametric formulation of Morlet wavelet is

$$
\psi(f, \zeta, \tau, t)=\psi_{\gamma}(t)=e^{\left(-\zeta / \sqrt{1-\zeta^{2}}\right)[2 \pi f(t-\tau)]^{2}} \cos (2 \pi f(t-\tau)),
$$

where the parameter vector $\gamma=(f, \zeta, \tau)$ determines the wavelet properties. These parameters $(f, \zeta, \tau)$ are denoted by frequency $f \in \mathbf{R}^{+}$, damping ratio $\zeta \in[0,1) \subset \mathbf{R}^{+}$and time index $\tau \in \mathbf{R}$, respectively.

The discrete parameters $f, \zeta$, and $\tau$ belong to subsets of $\mathbf{F}, \mathbf{Z}$, and $\mathbf{T}_{\mathbf{C}}$, respectively:

$$
\begin{gathered}
\mathbf{F}=\left\{f_{1}, f_{2}, \ldots, f_{m}\right\} \subset \mathbf{R}^{+}, \\
\mathbf{Z}=\left\{\zeta_{1}, \zeta_{2}, \ldots, \zeta_{n}\right\} \subset \mathbf{R}^{+} \cap[0,1), \\
\mathbf{T}_{\mathbf{C}}=\left\{\tau_{1}, \tau_{2}, \ldots, \tau_{p}\right\} \subset \mathbf{R} .
\end{gathered}
$$

The discrete grid $\mathbf{\Gamma}=\mathbf{F} \times \mathbf{Z} \times \mathbf{T}_{\mathbf{C}}$ is constructed, and the set of the Morlet Wavelet, whose parameters are contained in subsets of $\mathbf{F}, \mathbf{Z}$, and $\mathbf{T}_{\mathbf{C}}$, is called the dictionary shown as follows:

$\Psi=\left\{\psi_{\gamma}(t): \gamma \in \boldsymbol{\Gamma}\right\}=\left\{\psi(f, \zeta, \tau, t): f \in \mathbf{F}, \zeta \in \mathbf{Z}, \tau \in \mathbf{T}_{\mathbf{C}}\right\}$,

and each item in the dictionary is called an atom.

2.2. Correlation Filtering (CF). Correlation between two signals describes their similarity to each other or, in general term, their interrelationship. The degree of similarity between two real certain signals with limited energy, $\psi_{\gamma}(t)$ and $x(t)$, can be measured in terms of a correlation coefficient, defined as [20]

$$
c_{x(t) \psi_{\gamma}(t)}=\frac{\left|C_{x(t) \psi_{\gamma}(t)}\right|}{\sigma_{x(t)} \sigma_{\psi_{\gamma}(t)}}=\frac{\left|\int(x(t)-\bar{x})\left(\psi_{\gamma}(t)-\bar{\psi}_{\gamma}\right) \mathrm{d} t\right|}{\sqrt{\int(x(t)-\bar{x})^{2} \mathrm{~d} t \int\left(\psi_{\gamma}(t)-\bar{\psi}_{\gamma}\right)^{2} \mathrm{~d} t}},
$$

where $\sigma_{x(t)}$ and $\sigma_{\psi_{\gamma}(t)}$ are the standard deviations of the vibration signal $x(t)$ and the atom $\psi_{\gamma}(t)$, respectively, and 
$C_{x(t) \psi_{y}(t)}$ is the covariance of $x(t)$ and $\psi_{\gamma}(t)$. In practice, the signal $x(t)$ and the atom $\psi_{\gamma}(t)$ are sampled as discrete values; thus, the correlation coefficient is estimated from the sampled data as

$$
c_{\gamma}=\frac{\left|\sum_{k=1}^{N}(x(k)-\bar{x})\left(\psi_{\gamma}(k)-\bar{\psi}_{\gamma}\right)\right|}{\sqrt{\sum_{k=1}^{N}(x(k)-\bar{x})^{2} \sum_{k=1}^{N}\left(\psi_{\gamma}(k)-\bar{\psi}_{\gamma}\right)^{2}}},
$$

where $N$ is the number of the data samples, and $\bar{x}$ and $\bar{\psi}_{\gamma}$ are the mean values of $x(k)$ and $\psi_{\gamma}(k)$, respectively. Because of the approximate zero mean property of the wavelet and the vibration signal, (6) can be described as

$$
c_{\gamma}=\frac{\left|\sum_{k=1}^{N} x(k) \psi_{\gamma}(k)\right|}{\sqrt{\sum_{k=1}^{N} x^{2}(k) \sum_{k=1}^{N} \psi_{\gamma}^{2}(k)}}
$$

where $c_{\gamma}$ is a multidimensional matrix, which is determined by $\boldsymbol{\Gamma}=\mathbf{F} \times \mathbf{Z} \times \mathbf{T}_{\mathbf{C}}$. A correlation coefficient $k_{\gamma}(\tau)$ is defined for modal analysis to correlate frequency and damping at each time value. Peaks of $c_{\gamma}$ for a given $\tau$ relate the wavelet with the strongest correlation to the signal. Define $k_{\gamma}(\tau)$ as the peak values of $c_{\gamma}$ at each $\tau$. So, the formulation of $k_{\gamma}(\tau)$ is

$$
k_{\gamma}(\tau)=\max _{f \in \mathbf{F}, \zeta \in \mathbf{Z}} c_{\gamma}=c_{\{\bar{f}, \bar{\zeta}, \tau\}}
$$

where $\bar{f}$ and $\bar{\zeta}$ are the characteristic parameters of the Morlet wavelet associated with the peak correlation. Define

$$
k_{\gamma, \max }=\max _{\tau \in \mathbf{T}_{\mathbf{C}}} k_{\gamma}(\tau)=\max _{f \in \mathbf{F}, \zeta \in \mathbf{Z}, \tau \in \mathbf{T}_{\mathbf{C}}} c_{\gamma}=c_{\{\overline{f, \zeta, \bar{\tau}\}},}
$$

where $\bar{\tau}$ is the time of the peak value of $c_{\gamma}$ in the whole time domain.

2.3. Cyclic Morlet Wavelet Correlation Filtering (CMWCF). According to the characteristics of vibration signal and the identified Morlet wavelet $\psi_{\bar{\gamma}}(t)$ through $\mathrm{CF}$, in order to determine the period, that is, time interval between two adjacent impulse responses, we can define the cyclic Morlet wavelet by introducing parameter $T$ as

$$
\begin{aligned}
\varphi_{\lambda}(t) & =\sum_{k} \psi_{\gamma}(t-k T) \\
& =\sum_{k} A e^{\left(-\zeta / \sqrt{1-\zeta^{2}}\right)[2 \pi f(t-k T-\tau)]^{2}} \cos (2 \pi f(t-k T-\tau)),
\end{aligned}
$$

where $T$ is the time interval between two adjacent cyclic Morlet wavelet atoms, named cyclic period. Then, the parameter vector $\lambda=(f, \zeta, \tau, T)$ determines the cyclic Morlet wavelet properties.

According to the characteristics of cyclic impulse response, making use of the impulse response parameters which is obtained from CF, the cyclic Morlet wavelet is constructed to detect cyclic period. The set of the cyclic Morlet wavelet, whose parameter is contained in the subset of $\mathbf{T}_{\mathbf{T}}=\left\{T_{1}, T_{2}, \ldots, T_{q}\right\} \subset \mathbf{R}^{+}$, is also called the dictionary shown as

$$
\Phi=\left\{\varphi_{T}(t): T \in \mathbf{T}_{\mathbf{T}}\right\}=\left\{\varphi(\bar{f}, \bar{\zeta}, \bar{\tau}, T, t): T \in \mathbf{T}_{\mathbf{T}}\right\}
$$

where $\bar{f}, \bar{\zeta}$, and $\bar{\tau}$ are the parameters of impulse response obtained from CF. Then, a correlation function $k_{T}(T)$ is defined to quantify the correlation degree between $\varphi_{T}(t)$ and $x(t)$ :

$$
k_{T}(T)=\frac{\left|\sum_{k=1}^{N} x(k) \varphi_{T}(k)\right|}{\sqrt{\sum_{k=1}^{N} x^{2}(k) \sum_{k=1}^{N} \varphi_{T}^{2}(k)}} .
$$

Practically, $k_{T}(T)$ is a column vector, whose size is determined by subset of $\mathrm{T}_{\mathrm{T}}$. Define

$$
k_{T, \max }=\max _{T \in \mathbf{T}_{\mathrm{T}}} k_{T}(T)=k_{\{\overline{f, \zeta, \bar{\tau}, \bar{T}\}},}
$$

where $\bar{T}$, associated with the maximum of $k_{T}$, is cyclic period.

Then, both the parameters of Morlet wavelet and the cyclic period between adjacent wavelet atoms are identified. These identified parameters are associated with the impulse responses. Finally, the procedure of the adaptive parameter identification scheme proposed is summarized as follows:

(i) establish Morlet wavelet dictionary;

(ii) find optimal Morlet wavelet using correlation filtering based on maximal correlation coefficient criterion;

(iii) construct cyclic Morlet wavelet given by (10) obtained in step 2;

(iv) find cyclic period using CMWCF based on maximal correlation coefficient criterion.

\section{Simulation Signal Test}

A simulation study is performed to illustrate the effect of the CMWCF method. Consider a simulative signal

$$
\begin{aligned}
x(t)= & \sum_{k} \psi_{\gamma}(t-k T)+A_{n} n(t) \\
= & \sum_{k} e^{\left(-\zeta_{0} / \sqrt{1-\zeta_{0}^{2}}\right)\left[2 \pi f_{0}\left(t-k T_{0}-\tau_{0}\right)\right]^{2}} \cos \left(2 \pi f\left(t-k T_{0}-\tau_{0}\right)\right) \\
& +A_{n} n(t),
\end{aligned}
$$

where the frequency $f_{0}=5 \mathrm{~Hz}$, the damping ratio $\zeta_{0}=0.01$, the time index $\tau_{0}=1 \mathrm{~s}$, and the cyclic period $T_{0}=2 \mathrm{~s}$. Obviously, $x(t)$ is a real periodic cyclic impulse responses signal. The signal $n(t)$ is white noise weight by $A_{n}=0.2$, and the sampling frequency is $200 \mathrm{~Hz}$ in time range $[0,10]$.

The Morlet wavelet dictionary is adopted to analyze the simulation signal. The grid of wavelet parameters is determined according to the subsets of $\mathbf{F}=\{4.5: 0.01$ : $5.5\}, Z=\{\{0.005: 0.001: 0.02\} \cup\{0.03: 0.05: 0.9\}\}$, $\mathbf{T}_{\mathbf{C}}=\{0: 0.01: 10\}$, and $\mathbf{T}_{\mathbf{T}}=\{0.5: 0.005: 5\}$, where 
TABLE 1: The results of CMWCF when increasing the noise amplitude.

\begin{tabular}{lccccccc}
\hline$A_{n}$ & SNR $(\mathrm{dB})$ & $k_{\gamma, \max }$ & $\bar{f}(\mathrm{~Hz})$ & $\bar{\zeta}$ & $\bar{\tau}(\mathrm{s})$ & $k_{T, \max }$ & $\bar{T}(\mathrm{~s})$ \\
\hline 0 & $\infty$ & 0.4472 & 5.00 & 0.010 & 5.00 & 1.0000 & 2.000 \\
0.1 & 10.0120 & 0.4388 & 5.00 & 0.010 & 3.00 & 0.9522 & 2.000 \\
0.2 & 3.9053 & 0.3947 & 5.00 & 0.010 & 5.00 & 0.8454 & 2.000 \\
0.3 & 0.6009 & 0.3463 & 5.00 & 0.010 & 3.00 & 0.7427 & 2.000 \\
0.4 & -2.3101 & 0.2976 & 5.00 & 0.010 & 7.00 & 0.6253 & 2.000 \\
0.5 & -4.1144 & 0.2883 & 5.01 & 0.008 & 3.00 & 0.5378 & 2.000 \\
0.6 & -5.6627 & 0.2356 & 5.10 & 0.012 & 4.95 & 0.4167 & 2.000 \\
0.7 & -7.0026 & 0.2031 & 4.93 & 0.015 & 7.05 & 0.3846 & 1.995 \\
0.8 & -7.8508 & 0.2010 & 4.93 & 0.014 & 6.95 & 0.3676 & 1.995 \\
0.9 & -9.0012 & 0.1812 & 5.15 & 0.012 & 6.95 & 0.2688 & 2.010 \\
1.0 & -10.0057 & 0.1718 & 4.86 & 0.012 & 6.95 & 0.2851 & 1.990 \\
1.1 & -10.8197 & 0.1421 & 4.99 & 0.015 & 0.95 & 0.2802 & 2.000 \\
1.2 & -11.5463 & 0.1448 & 4.99 & 0.016 & 1.05 & 0.2695 & 2.000 \\
\hline
\end{tabular}

TABLE 2: Success rate for detecting the cyclic period.

\begin{tabular}{cccccc}
\hline$A_{n}$ & SNR $(\mathrm{dB})$ & Success rate & $A_{n}$ & SNR $(\mathrm{dB})$ & Success rate \\
\hline 0.1 & 10.0120 & $100 \%$ & 0.7 & -7.0026 & $85 \%$ \\
0.2 & 3.9053 & $96 \%$ & 0.8 & -7.8508 & $78 \%$ \\
0.3 & 0.6009 & $90 \%$ & 0.9 & -9.0012 & $78 \%$ \\
0.4 & -2.3101 & $95 \%$ & 1.0 & -10.0057 & $81 \%$ \\
0.5 & -4.1144 & $81 \%$ & 1.1 & -10.8197 & $80 \%$ \\
0.6 & -5.6627 & $92 \%$ & 1.2 & -11.5463 & $77 \%$ \\
\hline
\end{tabular}

$\mathbf{F}=\{4.5: 0.01: 5.5\}$ is a Matlab notation which denotes an array from 4.5 to 5.5 with step 0.01 , and $\mathbf{Z}, \mathbf{T}_{\mathrm{C}}$, and $\mathbf{T}_{\mathbf{T}}$ are similar to $\mathbf{F}$. The parameter subset of $\mathbf{Z}$ is nonuniform to provide higher resolution at lower damping ratio values. The results obtained by the proposed method from the simulation signal are shown in Figure 1.

Figure 1(a) gives the waveform of the simulation signal without noise and Figure 1(b) with noise. Figure 1(c) represents the correlation value $k_{\gamma}(\tau)$, whose peak value $k_{\gamma, \max }=0.3947$ locates at one impulse. Figures $1(\mathrm{~d})$ and $1(\mathrm{e})$ indicate the modal information of frequency and damping ratio parameters revealed from the peak correlation $k_{\gamma}(\tau)$ at each time $\tau$. We obtained frequency $\bar{f}=5 \mathrm{~Hz}$ and damping ratio $\bar{\zeta}=0.01$ which are exactly equal to simulation values $\left(f_{0}=5 \mathrm{~Hz}, \zeta_{0}=0.01\right)$. Because of the multi-impulse the time index $\bar{\tau}=5 \mathrm{~s}$ is not equal to $\tau_{0}$ shown in Figure 1(c). Using the results obtained by correlation filtering, the cyclic Morlet wavelet is constructed. Then, the correlation value $k_{T}(T)$, shown in Figure 1(f), is obtained by CMWCF, and the cyclic period $\bar{T}=2 \mathrm{~s}$ associated with $k_{T, \max }=0.8454$ is identified, which is also equal to the simulation value $\left(T_{0}=2 \mathrm{~s}\right)$. Figure $1(\mathrm{~g})$ gives the comparison between the reconstructed cyclic Morlet wavelet with the obtained results and the simulation signal. To see more clearly, we parallelly move the curve of the reconstructed impulse response. The consistency between them can be obviously seen, so it can be drawn that the proposed method is effective in identifying the cyclic period between adjacent impulses.

In order to test the noise tolerance of the method, the simulation test with different noise amplitudes $A_{n}$ from (14) is investigated shown in Table 1 , in which the results $A_{n}, \mathrm{SNR}$, $k_{\gamma, \text { max }}, \bar{f}, \bar{\zeta}, \bar{\tau}, k_{T, \max }$, and $\bar{T}$ are listed. SNR, the signal-tonoise ratio, is used to weigh the noise level and is defined as follows:

$$
\mathrm{SNR}=10 \times \log \left(\frac{P_{S}}{P_{N}}\right),
$$

where $P_{S}$ is the energy of the useful information and $P_{N}$ is the energy of the noise.

It is clear that, with the increase of noise amplitude, the correlation values $k_{\gamma, \max }$ and $k_{T, \max }$ decreased steadily, illustrating that the noise reduces the correlation between the simulation signal and Morlet wavelet. Meanwhile, the noise amplitude influences the frequency $\bar{f}$, the damping ration $\bar{\zeta}$, and the time $\bar{\tau}$ for $A_{n}>0.4$, but it has little influence on the cyclic period. Table 2 gives the success rate of detecting the cyclic period for randomized trial on 100 times.

\section{Application in Gearbox Fault Feature Detection}

To study the effectiveness of the presented methods for the gearbox fault feature detection, our experiment is concerned with a fatigue test of an automobile transmission gearbox. The structure of the gearbox is shown in Figure 2, which has five forward speeds and one backward speed. The vibration signal was acquired by an accelerometer mounted on the outer case of the gearbox when it is loaded with the third speed gearbox. 


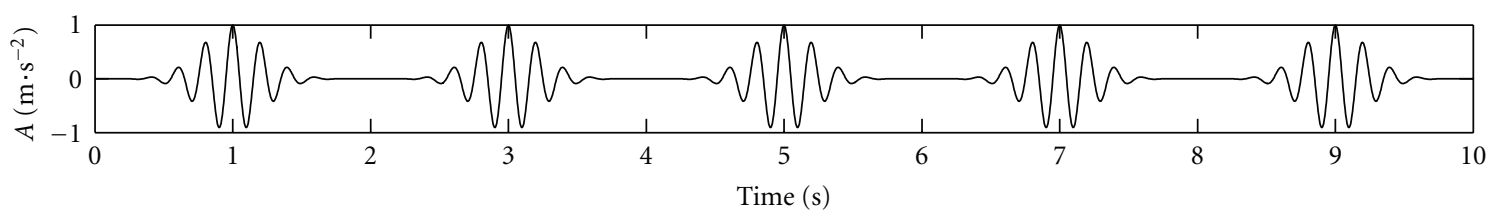

(a)

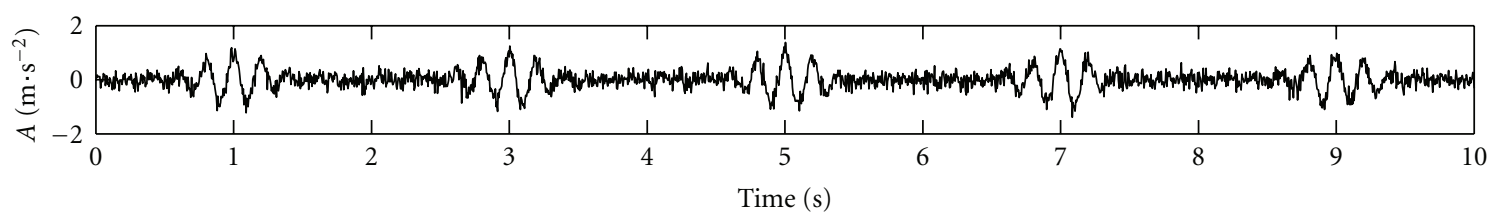

(b)

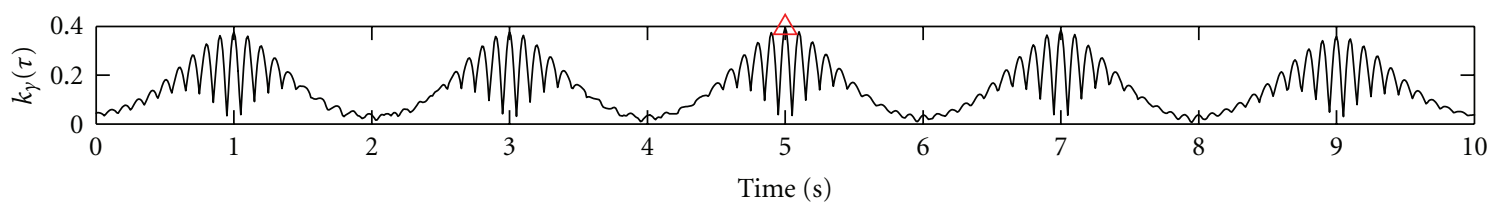

(c)

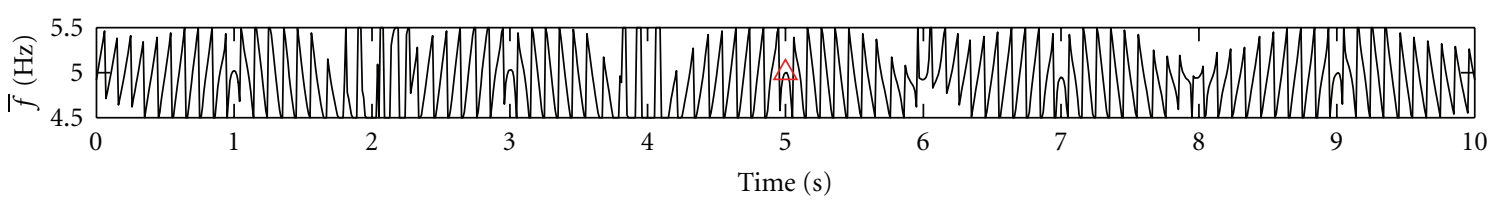

(d)

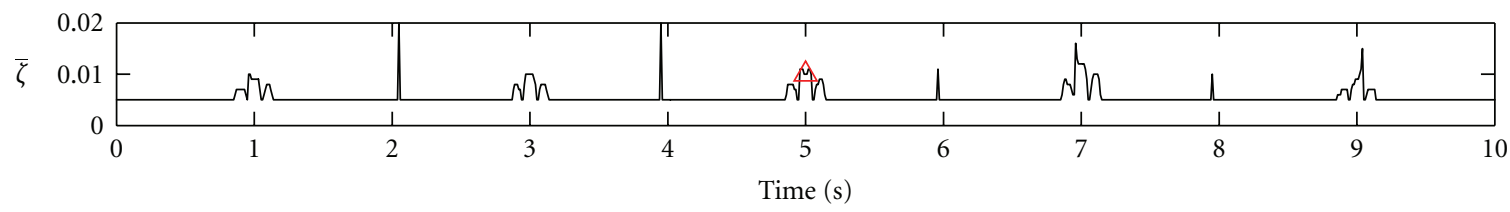

(e)

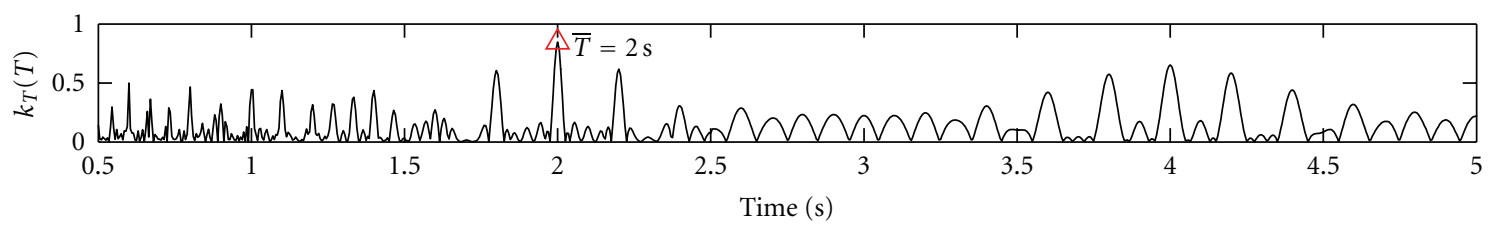

(f)

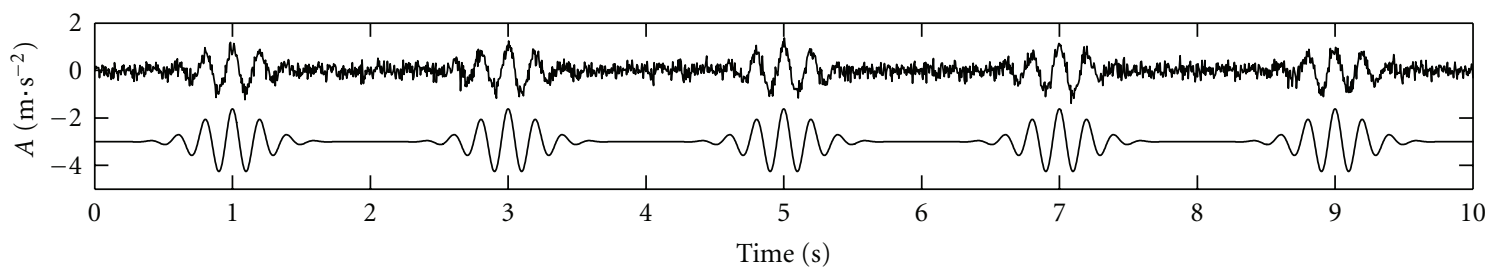

(g)

FIGURE 1: CMWCF of the simulation signal: (a) the simulation signal, (b) the simulation signal with noise, (c) the correlation value of CF, (d) frequency parameter $\bar{f}$, (e) damping ratio parameter $\bar{\zeta}$, (f) the correlation value of CMWCF, and (g) the comparison between the vibration signal and reconstructed signal.

For a gear transmission, the meshing frequency $f_{m}$ is calculated by

$$
f_{m}=\frac{n z}{(60 i)}
$$

where $z$ is the number of gear teeth, $n$ is the rotating speed of the input shaft, and $i$ is the transmission ratio. In the test, $z=$ $27, n=1600 \mathrm{rpm}$, and $i=1.44$. Then, the meshing frequency of the third speed is calculated to be $500 \mathrm{~Hz}$. The sampling 


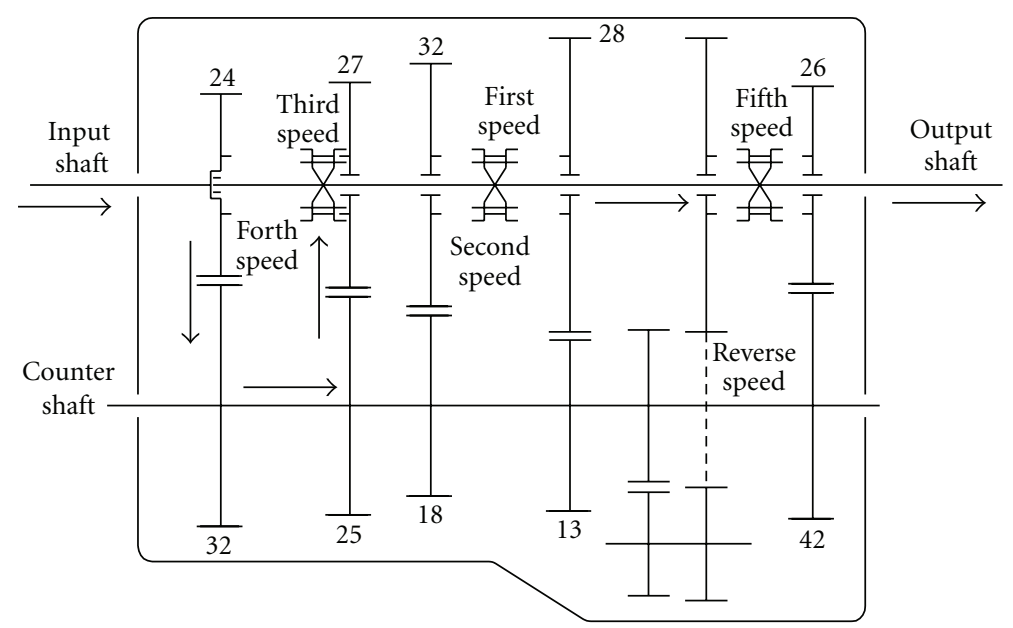

(a) Structure of the gearbox

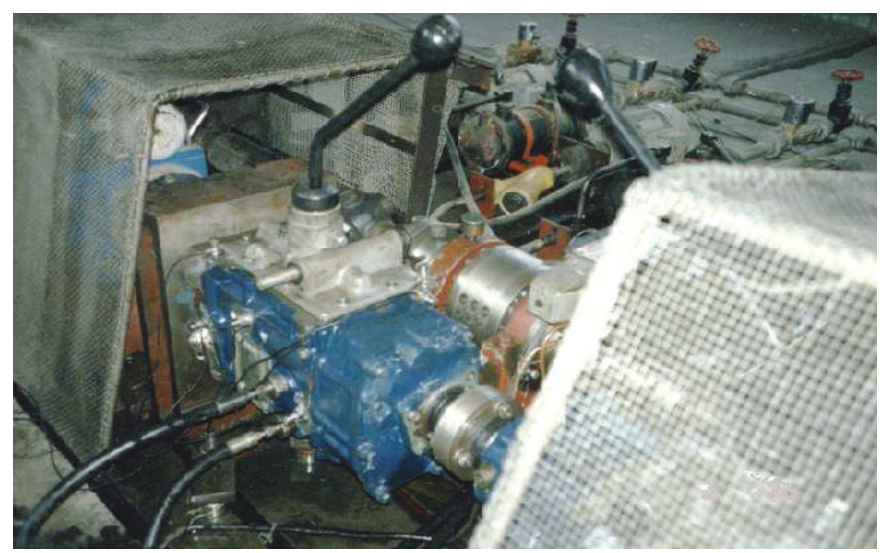

(b) Gearbox setup

FIgURE 2: The automobile transmission gearbox.

TABLE 3: Working parameters of the third speed gears.

\begin{tabular}{|c|c|c|c|c|}
\hline & \multicolumn{2}{|c|}{ The third speed gears } & \multicolumn{2}{|c|}{ Constant meshing gears } \\
\hline & Driving gear & Driven gear & Driving gear & Driven gear \\
\hline Number of teeth & 25 & 27 & 24 & 32 \\
\hline Rotating period (s) & 0.05 & 0.054 & 0.04 & 0.03 \\
\hline Rotating frequency $(\mathrm{Hz})$ & 20 & 18.5 & 25 & 33.3 \\
\hline Meshing frequency $(\mathrm{Hz})$ & \multicolumn{2}{|c|}{500} & \multicolumn{2}{|c|}{640} \\
\hline
\end{tabular}

frequency is $3 \mathrm{KHz}$. The working parameters are shown in Table 3.

The typical vibration signal caused by one driving gear teeth broken is shown in Figure 3(a). The time domain signal fails to demonstrate the characteristic feature of the gearbox vibration signal. Figure 3(b) expresses the corresponding frequency spectrum, from which it can be seen that the $500 \mathrm{~Hz}$ in frequency is the main components. Figure 3(c) gives the waveform of the correlation value $k_{\gamma}(\tau)$ which is the result of correlation filtering, in which the maximal correlation value $k_{\gamma, \max }=0.3370$ is marked. The associated parameters are $\bar{f}=268 \mathrm{~Hz}, \bar{\zeta}=0.0060$, and $\bar{\tau}=0.1780 \mathrm{~s}$.

In order to identify the cyclic period, the reconstructed Morlet wavelet is used to construct cyclic Morlet wavelet. The correlation coefficient $k_{T}$ between constructed cyclic Morlet wavelet and the vibration signal under different parameter period $T$ is given in Figure 3(d), in which the maximal correlation value $k_{T, \max }=0.5711$ is marked and the corresponding period is $\bar{T}=0.050 \mathrm{~s}$. The comparison between the reconstructed cyclic Morlet wavelet and the 


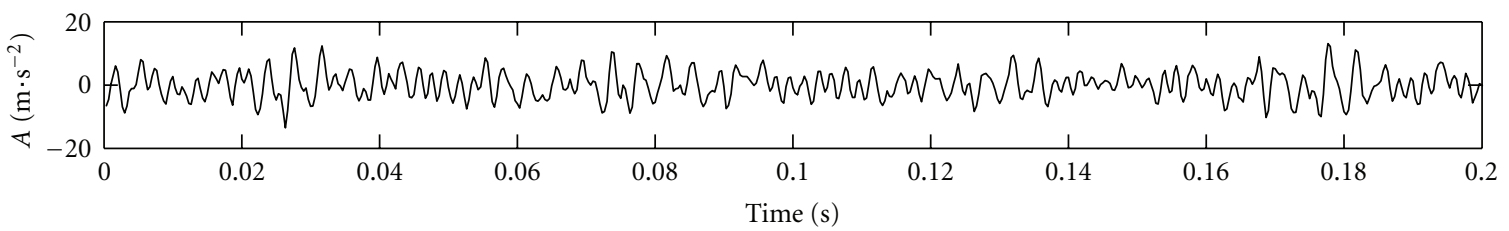

(a)

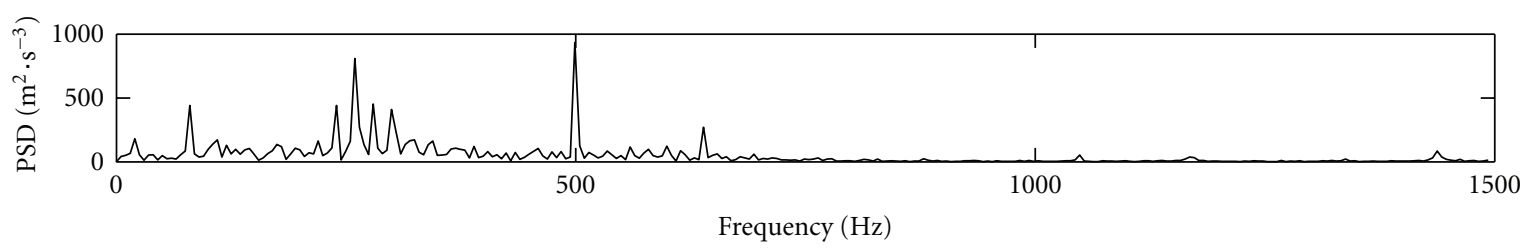

(b)

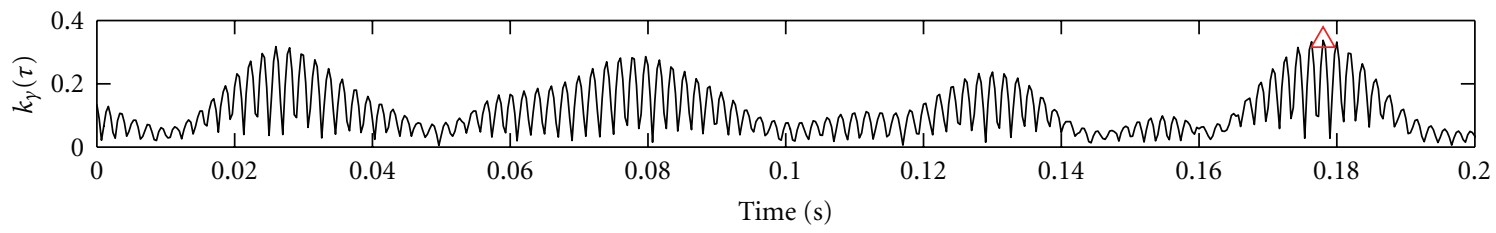

(c)

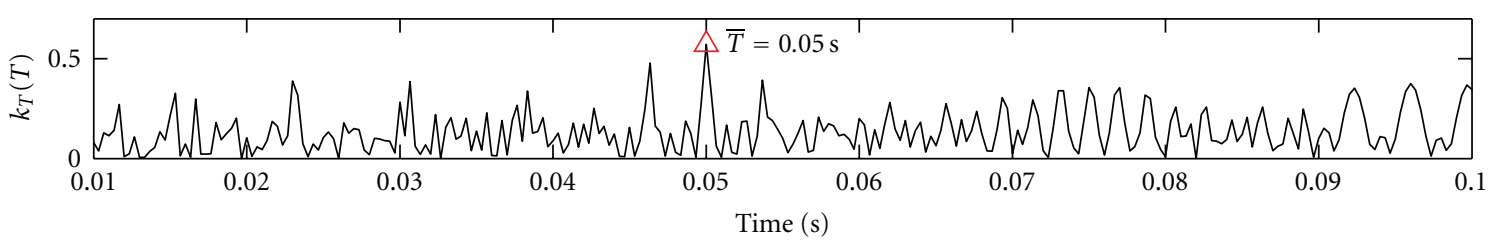

(d)

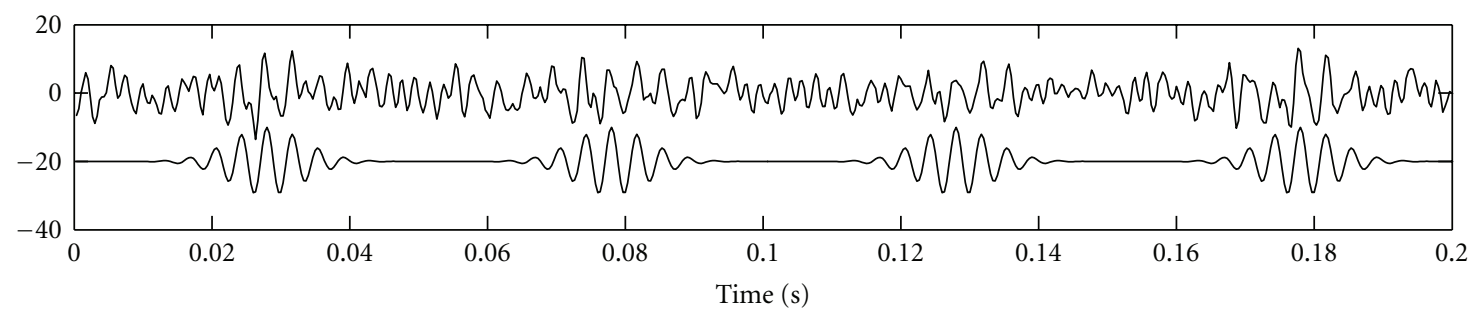

(e)

FIGURE 3: The CMWCF application in gearbox vibration: (a) the gearbox vibration signal, (b) the spectrum of the gearbox vibration, (c) the correlation value of CF, (d) the correlation value of CMWCF, and (e) the comparison of the vibration signal and reconstructed signal.

original vibration signal is given in Figure 3(e), in which, to see more clearly, the curve of the reconstructed one is also parallelly moved. Obviously, as shown in Table 2, the identified cyclic period is consistent with the rotating period of the third speed driving gear. That is to say, the proposed method is effective in identifying the characteristic parameters.

To prove the tolerance of the proposed method, Figure 4 gives another signal whose length is different and the results include $k_{\gamma, \max }=0.2877, \bar{f}=275 \mathrm{~Hz}, \bar{\zeta}=0.0080, \bar{\tau}=$ $0.2310 \mathrm{~s}, k_{T, \max }=0.4327$, and $\bar{T}=0.050 \mathrm{~s}$. These identified parameters are almost identical to the result of Figure 3.

Furthermore, in addition to illustrate the effectiveness of the proposed method, Figure 5 gives the vibration signal on normal condition, in which $k_{\gamma, \max }=0.1187$ and $k_{T, \max }=0.1236$ are represented and smaller than results of Figures 3 and 4. It is illustrated that there is no apparent impulse response in the vibration signal. 


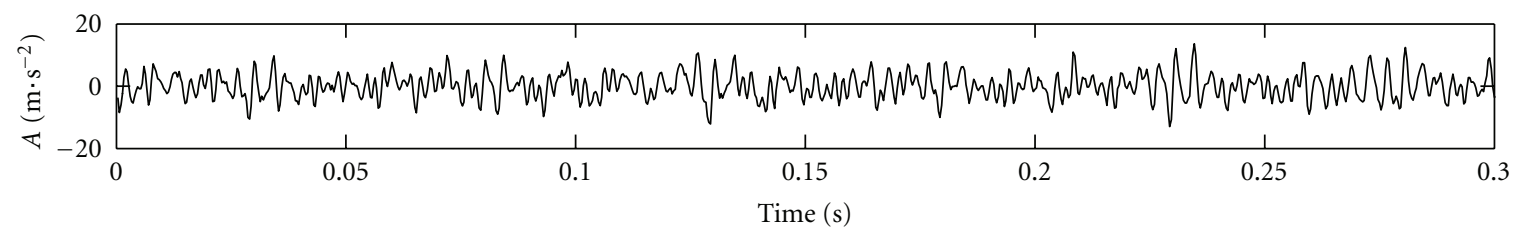

(a)

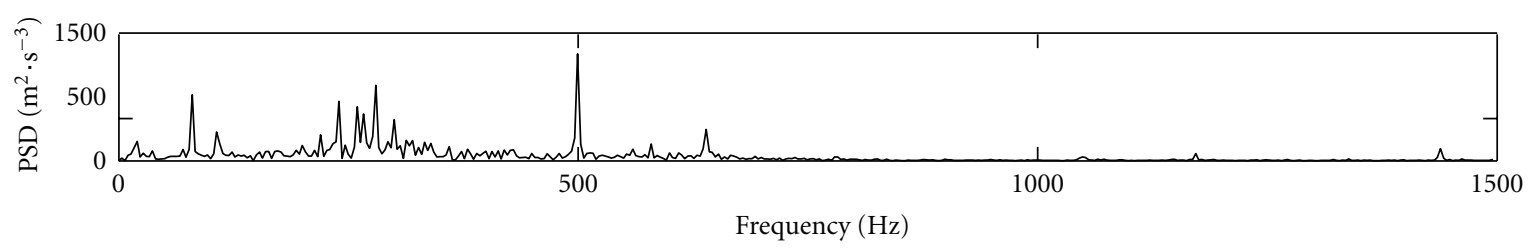

(b)

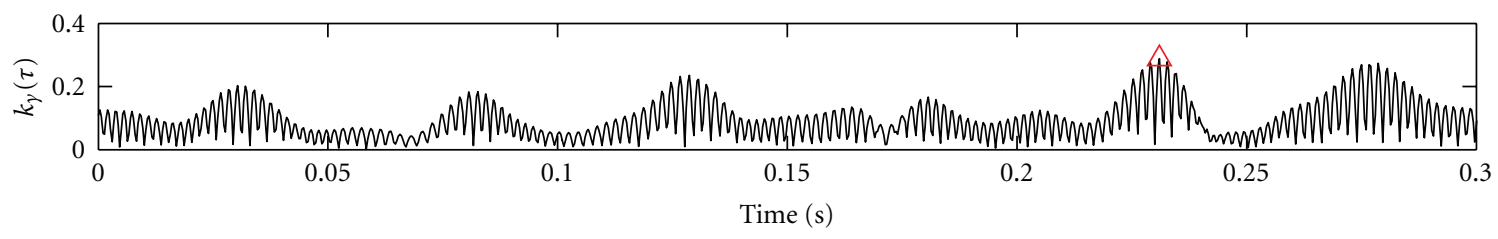

(c)

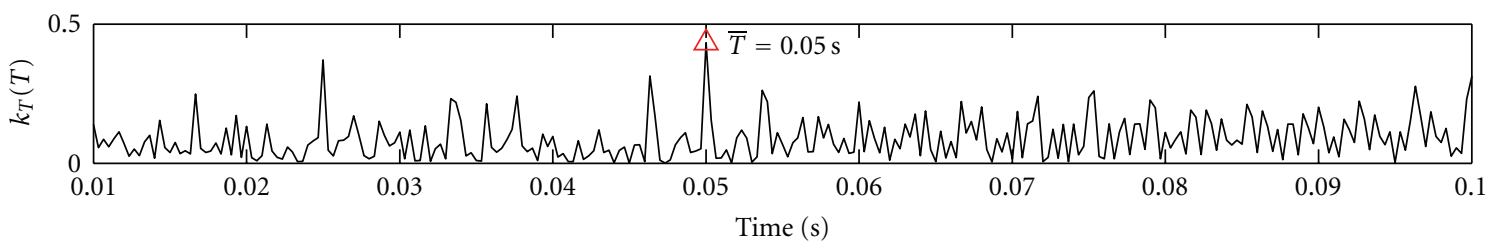

(d)

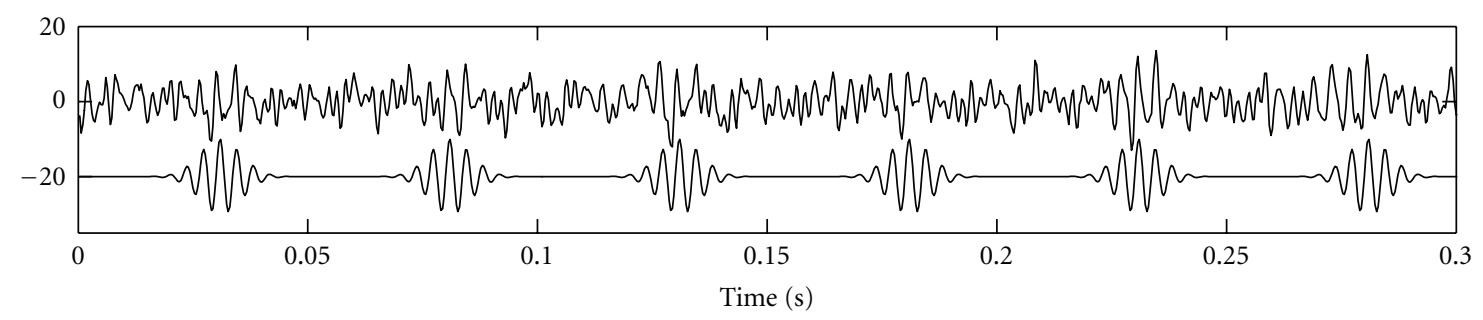

(e)

FIGURE 4: The CMWCF application in gearbox vibration: (a) the gearbox vibration signal, (b) the spectrum of the gearbox vibration, (c) the correlation value of CF, (d) the correlation value of CMWCF, and (e) the comparison of the vibration signal and reconstructed signal.

Though the impulse response was reconstructed and given in Figure 5(e), it can be clearly observed that the comparison with the original signal is unaccountable. So it can be drawn that there is no cyclic impulse response in the vibration signal on normal condition. In other words, it can be drawn that there is no localized defect in the gearbox, and this also verifies the effectiveness of the proposed method. 


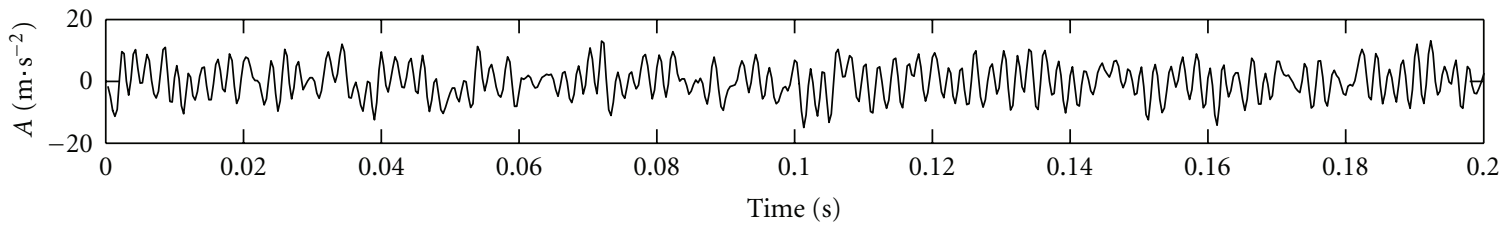

(a)

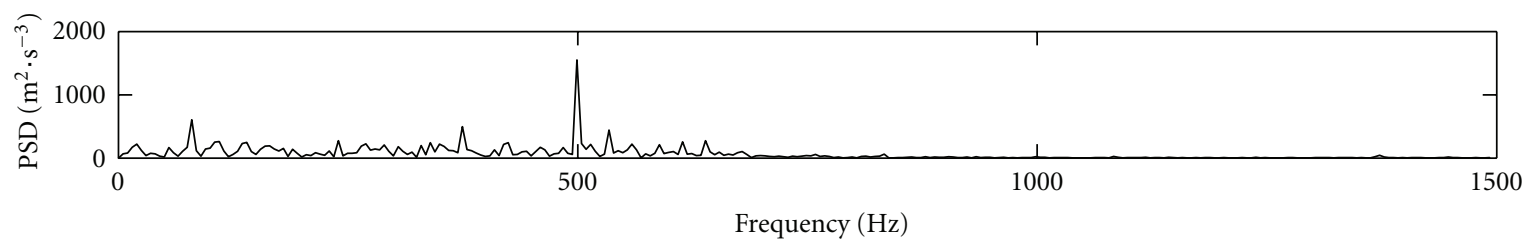

(b)

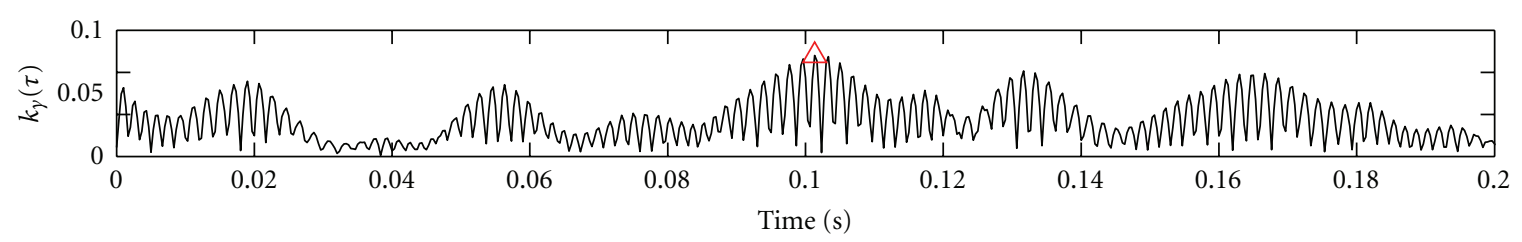

(c)

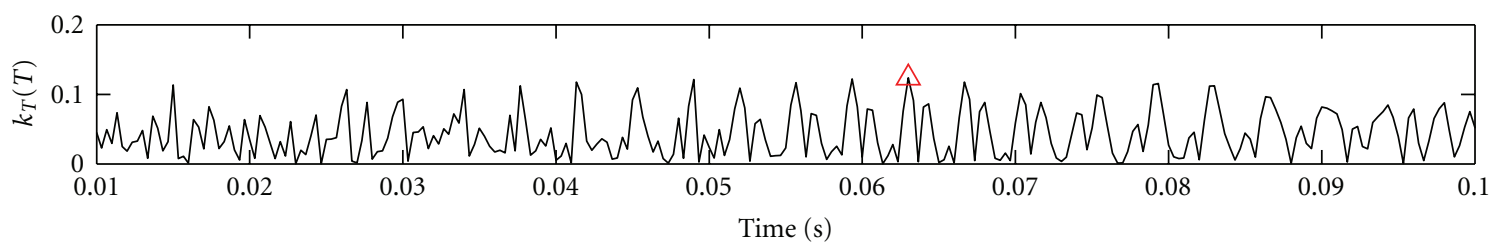

(d)

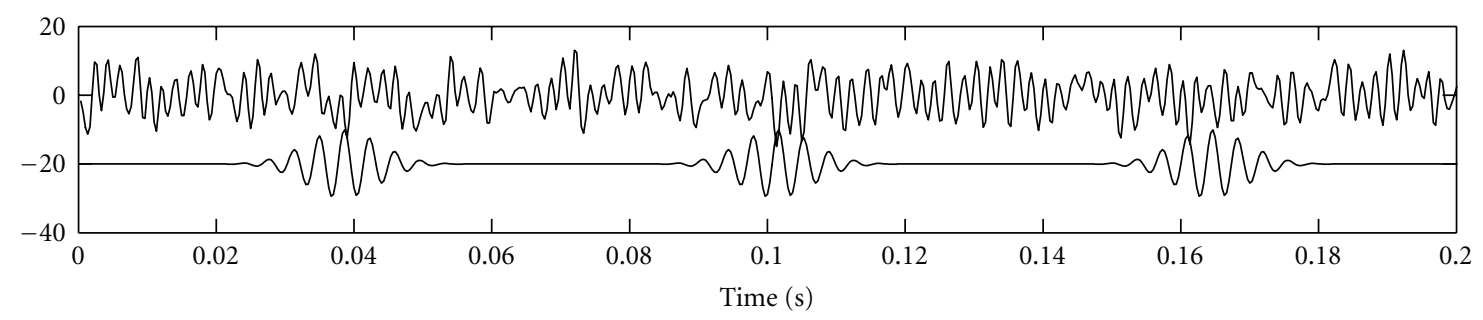

(e)

FIGURE 5: The CMWCF application in gearbox vibration: (a) the gearbox vibration signal, (b) the spectrum of the gearbox vibration, (c) the correlation value of CF, (d) the correlation value of CMWCF, and (e) the comparison of the vibration signal and reconstructed signal.

\section{Conclusions}

The cyclic Morlet wavelet correlation filtering (CMWCF) method proposed represents an attempt in the direction of parameter identification and feature detection for fault diagnosis. Both the parameters of the Morlet wavelet associated with the maximal correlation value and the cyclic period are effective in feature detection of the impulse response.

The simulation study demonstrates that the proposed method is effective in identifying parameters of impulse, including frequency, damping ratio, and the time index, and is especially sensitive to the cyclic period. The gearbox application also demonstrates the fact that the method has the capability of parameter identification.

In conclusion, the other gearbox applications have not yet been provided in the paper; however, it conforms that CMWCF provides a feature detection method for gearbox fault diagnosis. Furthermore, the method has the potential applicability for monitoring other rotating mechanical components such as bearings and rotors. 


\section{Acknowledgments}

This research is supported partly by the Natural Science Foundation of China (no. 50905021) and the Natural Science Foundation of Jiangsu Province (no. BK2010225).

\section{References}

[1] Z. Feng, M. J. Zuo, and F. Chu, "Application of regularization dimension to gear damage assessment," Mechanical Systems and Signal Processing, vol. 24, no. 4, pp. 1081-1098, 2010.

[2] P. D. Samuel and D. J. Pines, "A review of vibration-based techniques for helicopter transmission diagnostics," Journal of Sound and Vibration, vol. 282, no. 1-2, pp. 475-508, 2005.

[3] B. Leprettre and N. Martin, "Extraction of pertinent subsets from time-frequency representations for detection and recognition purposes," Signal Processing, vol. 82, no. 2, pp. 229-238, 2002.

[4] M. Blödt, M. Chabert, J. Regnier, and J. Faucher, "Mechanical load fault detection in induction motors by stator current time-frequency analysis," IEEE Transactions on Industry Applications, vol. 42, no. 6, pp. 1454-1463, 2006.

[5] E. G. Strangas, S. Aviyente, and S. S. H. Zaidi, "Timefrequency analysis for efficient fault diagnosis and failure prognosis for interior permanent-magnet AC motors," IEEE Transactions on Industrial Electronics, vol. 55, no. 12, pp. 41914199, 2008.

[6] Z. K. Peng and F. L. Chu, "Application of the wavelet transform in machine condition monitoring and fault diagnostics: a review with bibliography," Mechanical Systems and Signal Processing, vol. 18, no. 2, pp. 199-221, 2004.

[7] N. E. Huang, Z. Shen, S. R. Long et al., "The empirical mode decomposition and the Hubert spectrum for nonlinear and non-stationary time series analysis," Proceedings of the Royal Society A, vol. 454, no. 1971, pp. 903-995, 1998.

[8] Y. Wang, Z. He, and Y. Zi, "Enhancement of signal denoising and multiple fault signatures detecting in rotating machinery using dual-tree complex wavelet transform," Mechanical Systems and Signal Processing, vol. 24, no. 1, pp. 119-137, 2010.

[9] Q. Gao, C. Duan, H. Fan, and Q. Meng, "Rotating machine fault diagnosis using empirical mode decomposition," Mechanical Systems and Signal Processing, vol. 22, no. 5, pp. 1072-1081, 2008.

[10] Y. Lei, Z. He, and Y. Zi, "Application of the EEMD method to rotor fault diagnosis of rotating machinery," Mechanical Systems and Signal Processing, vol. 23, no. 4, pp. 1327-1338, 2009.

[11] S. G. Mallat and Z. Zhang, "Matching pursuits with timefrequency dictionaries," IEEE Transactions on Signal Processing, vol. 41, no. 12, pp. 3397-3415, 1993.

[12] L. C. Freudinger, R. Lind, and M. J. Brenner, "Correlation filtering of modal dynamic using the Laplace wavelet," in Proceedings of the 16th International Modal Analysis Conference (IMAC '98), vol. 2, pp. 868-877, Santa Barbara, Calif, USA, February 1998.

[13] Y. Zi, X. Chen, Z. He, and P. Chen, "Vibration based modal parameters identification and wear fault diagnosis using Laplace wavelet," Key Engineering Materials, vol. 293-294, pp. 183-190, 2005.

[14] K. Qi, J. Xiang, Y. Zi, and Z. He, "High precision modal parameter identification based on Laplace wavelet correlation filtering," Chinese Journal of Mechanical Engineering, vol. 43, no. 9, pp. 167-172, 2007.
[15] H. B. Dong, X. F. Chen, B. Li, K. Y. Qi, and Z. J. He, "Rotor crack detection based on high-precision modal parameter identification method and wavelet finite element model," Mechanical Systems and Signal Processing, vol. 23, no. 3, pp. 869-883, 2009.

[16] J. Lin and L. Qu, "Feature extraction based on morlet wavelet and its application for mechanical fault diagnosis," Journal of Sound and Vibration, vol. 234, no. 1, pp. 135-148, 2000.

[17] J. Lin and M. J. Zuo, "Gearbox fault diagnosis using adaptive wavelet filter," Mechanical Systems and Signal Processing, vol. 17, no. 6, pp. 1259-1269, 2003.

[18] Z. K. Zhu, Z. H. Feng, and F. R. Kong, "Cyclostationarity analysis for gearbox condition monitoring: approaches and effectiveness," Mechanical Systems and Signal Processing, vol. 19, no. 3, pp. 467-482, 2005.

[19] A. Raad, J. Antoni, and M. Sidahmed, "Indicators of cyclostationarity: theory and application to gear fault monitoring," Mechanical Systems and Signal Processing, vol. 22, no. 3, pp. 574-587, 2008.

[20] J. S. Bendat and A. G. Piersol, Random Data: Analysis and Measurement Procedures, John Wiley \& Sons, New York, NY, USA, 3rd edition, 2000. 